\title{
Minimal clinically significant difference in health status: the thorny path of health status measures?
}

\author{
T. Hajiro*, K. Nishimura\#
}

Health status or health-related quality of life is being recognized as an important outcome when evaluating patients with chronic pulmonary disease. There is growing literature about health status measures in patients with asthma and chronic obstructive pulmonary disease (COPD). The European Respiratory Journal (ERJ) has published numerous articles on issues related to health status. The authors believe that the ERJ is a valuable resource in this area, especially for summarizing updated issues on the health status of pulmonary disease [1], or validating new questionnaires or comparing health status measures.

Clinicians need to understand and critically evaluate measurements of health status in order to determine if a new therapy could be applied to their own patients with expectation of improving their health status. However, it is also true that many readers face confusion and difficulty in interpreting the results of health status measures. Important concepts when evaluating measurements of health status are the clinically significant threshold or the minimal clinically significant difference (MCSD) [2]. When health status is measured using a continuous scale, it needs to be known whether an observed difference indicates a clinically significant or trivial effect on the patients' health status or quality of life. A statistically significant difference in health status might be of little practical importance; it is more important to know the MCSD. There are several definitions of the MCSD that correspond to the perspective of those who evaluate. For example, JAESCHKE et al. [3] define "minimal clinically important difference" as the smallest difference in a score in a domain of interest that patients perceive as beneficial and that would mandate, in the absence of side-effects and a change in the patient's management [3].

In the current issue of the ERJ, JONES [4] has analysed three representative health status measures (Asthma Quality of Life Questionnaire (AQLQ), Chronic Respiratory Questionnaire (CRQ) and St George's Respiratory Questionnaire (SGRQ)) for asthma and COPD. This article is an important contribution not only to the MCSD for these

\footnotetext{
*Dept of Pulmonary Diseases, Kobe Nishi City Hospital, Kobe, and ${ }^{\#}$ Respiratory Division, Kyoto-Katsura Hospital, Kyoto, Japan.

Correspondence: T. Hajiro, Dept of Pulmonary Diseases, Kobe Nishi City Hospital, 2-4, Ichiban-cho, Nagata, Kobe, Japan. Fax: 81 785765358. E-mail:thaj@msi.biglobe.ne.jp
}

measures of asthma and COPD, but also to the clinical significance of many important parameters used in pulmonary medicine.

However, as JONES [4] describes, there are difficulties and complexities with methods to determine the MCSD. Since there is no "gold standard" for measuring health status, the subjective evaluation of "clinically significant" becomes important for health status [3]; that is, health status measures may be compared or anchored to other clinical changes or results, which is called anchor-based interpretation. When patient judgement is relied on, patients are asked how they "feel" about the effect of treatment (within-patient methods) or about their health, relative to that of their healthy counterparts (betweenpatient methods). The most commonly used anchorbased interpretation is based on a patient global rating question. For example, REDELMEIER et al. [5] selected the patients' response of a "little bit better or worse" to be equivalent to the minimal important difference in their study. However, there would be no way to validate the arbitrary choice of a "little bit better or worse" on a 5-point global scale representing the minimal important difference. The reliability of patient judgement of their health remains problematic, and the relevant anchor, judged clinically significant, may differ within different patient groups. Some studies have suggested that the patients' judgement be used as an interim "gold standard", although results to date do not support this suggestion. Health status needs to be evaluated, largely because patients' self-judgement does not correlate well with scores of health status measures. Therefore, while there may be some validity in relating changes in health status scores (e.g. MCSD) to a patient's perception of change, the limitations with this method must be highlighted.

Another anchor-based interpretation is based on the clinicians' perception. It is possible for clinicians to provide judgements when changes in scores are clinically significant. For example, in a study using the Functional Status Questionnaire, the researchers presented different sets of item responses that would result in specific scores to clinicians. The clinicians were asked to decide scores that they thought clinically significant or important [6]. However, there is substantial data showing that the patients' perspective and the clinicians' perspective usually differ.

The concept of "criterion referencing" may shed more light on how the MCSD of health status 
measures are evaluated. Criteria are created for events that clearly signal a worsened health status, such as hospital admission, or death, or a need for a major change in treatment. Although these criteria are selected arbitrarily, the method is reasonable since events such as hospital admission or death clearly show the jeopardized health status and should be reflected by worse scores of health status measures. JONES [4] quoted two studies on this subject and came to the conclusion that the minimal significant difference was approximately 4 units on the SGRQ. Analyses like these could also be performed for the CRQ and AQLQ in order to determine and confirm their minimal significant difference. In patients with asthma, it would be of interest to determine the variation or discrepancy in AQLQ scores between patients who have frequent hospital admissions due to acute asthma and those who do not.

Although there are no standard or universally accepted ways for defining or determining the MCSD, the fact that different approaches for evaluating the MCSD have reached similar conclusions suggests that a score of 0.5 for the CRQ and AQLQ, and a score of 4 for the SGRQ should be used as the interim standards for the clinical thresholds.

Once the minimal significant difference for health status measures is understood, the next step is to interpret score changes in measures. JONES [4] suggested three possible techniques: 1) comparing the change with average estimate for the clinical threshold; 2) determining number of patients whose change in score exceeded the threshold; and 3) judging the therapeutic response using the confidential interval method. The number needed to treat (NNT) is calculated as the reciprocal of the absolute risk reduction, and has been widely used to proxy for the relative risk reduction in the field of evidence-based medicine. The finding by JonEs [4] of a weak relationship between the MCSD and the NNT is surprising. Further studies, however, may be warranted in order to clarify whether or not the NNT is related to "the exact value of the threshold for clinical significance".

Some important points should be mentioned regarding the MCSD. First, the MCSD is important not only for health status measures, but also for other clinical parameters such as the forced expiratory volume in one second (FEV1), 6 min walk distance (6MW), and peak expiratory flow rates. REDELMEIER and co-workers [6, 7] adopted what they called "subjective comparison ratings" when they identified clinically significant thresholds of the FEV1 and $6 \mathrm{MW}$. Patients were asked to consider how other patients with COPD were doing and given a question like "compared to this person, my ability to walk is $X$ ?" Additional studies are required to determine if the MCSD of the FEV1 or $6 \mathrm{MW}$ can be determined by patient judgement. Criterion judgements can also be used to explore the MCSD of the FEV1. For example, the FEV1 of patients with COPD who have experienced acute exacerbations or death could be compared with the FEV1 of patients who have not.

Second, do patients with different health status scores have the same MCSD? It may be that patients with very mild disturbances in health status show different MCSDs compared with those with severe disturbances in health status. For example, consider two hypothetical patients, patient A with an SGRQ total score of 20 and patient B with an SGRQ total score 80 . When a bronchodilator is given to both patients, both experience a substantial benefit and the SGRQ score is reduced by 4 , which is equivalent to the MCSD. Does this mean that both patients experience the same level or impact of benefit to their health status? Jones [4] illustrated five patients under different scenarios, suggesting that even patients who have different levels of health status disturbance may share similar MCSDs.

The article by JONES [4] offers clear guidance on clinical thresholds for evaluating changes in health status measures. The scores presented can be regarded as standard values for the minimal clinically significant difference; this information will be useful when the results of clinical studies are critically assessed. Although the difficulty and complexity in defining and determining the minimal clinically significant difference should be pointed out, health status measures should be established as mandatory clinical parameters in pulmonary medicine.

\section{References}

1. European Respiratory Society. Quality of life in the failing lung. Eur Respir Rev 1997; 7: 42-104.

2. Wright JG. The minimal important difference: who's to say what is important? J Clin Epidemiol 1996; 49: 1221-1222.

3. Jaeschke R, Singer J, Guyatt GH. Measurement of health status. Ascertaining the minimal clinically important difference. Controll Clin Trials 1989; 10: 407-415.

4. Jones PW. Interpreting thresholds for a clinically significant change in health status in asthma and COPD. Eur Respir J 2002; 19: 398-404.

5. Redelmeier DA, Guyatt GH, Goldstein RS. Assessing the minimal important difference in symptoms: a comparison of two techniques. J Clin Epidemiol 1996; 49: 1215-1219.

6. Redelmeier DA, Goldstein RS, Min ST, Hyland RH. Spirometry and dyspnea in patients with COPD. Chest 1996; 109: 1163-1168.

7. Redelmeier DA, Bayoumi AM, Goldstein RS, Guyatt GH. Interpreting small differences in functional status: the six minute walk test in chronic lung disease patients. Am J Respir Crit Care Med 1997; 155: 12781282. 